\title{
Experimental observations of Soret-driven convection in the transient diffusive boundary layer
}

\author{
Stephan Messlinger, Christoph Kramer, Jürgen J. Schmied, ${ }^{*}$ Florian Winkel, ${ }^{\dagger}$ Wolfgang Schöpf, and Ingo Rehberg \\ Experimentalphysik V, Universität Bayreuth, 95440 Bayreuth, Germany
}

(Received 15 September 2013; published 25 November 2013)

\begin{abstract}
The onset of transient Soret-driven convection is investigated experimentally in a colloidal suspension of thermosensitive nanoparticles by the shadowgraph technique and by particle tracking observations. From the shadowgraph images, the concentration profile is reconstructed, giving evidence of a convective motion inside the transient boundary layer. Furthermore, the latency times for the convection onset are extracted from the measurements. The results point out that particle tracking is superior to the shadowgraph method for detecting the onset of convection. The onset latency times obtained from these experiments obey scaling laws which are in accordance with the predictions from theoretical treatments.
\end{abstract}

DOI: 10.1103/PhysRevE.88.053019

PACS number(s): 47.20.Bp, 47.55.pb, 47.55.pd

\section{INTRODUCTION}

When an initially motionless fluid layer is heated from below, the heating creates a density gradient with denser fluid being stratified above less dense fluid, which is due to the thermal expansion of the fluid. If the temperature difference between bottom and top is increased quasistatically, i.e., slow enough for the system to develop a linear, stationary temperature profile at any given time, a convective motion in the form of a pattern of parallel vortex rolls sets in once the density gradient exceeds a certain critical value [1]. This behavior is well understood theoretically by a linear stability analysis of the motionless basic state and is called RayleighBénard convection. This system has been studied extensively in the linear and nonlinear regime, both experimentally and theoretically [2-5].

If the temperature difference is increased instantly rather than quasistatically, the temperature distribution inside the fluid will transiently deviate from the linear profile. Instead, it develops a diffusive profile with steep density gradients near the boundaries (so-called diffusive boundary layers). Once the density stratification inside such a layer reaches an unstable state, convection will start locally inside the boundary layer. Depending on the temperature difference, this transient convective motion may start long before a stationary profile is established over the complete layer height.

Such transient effects are of particular importance for convection driven by thermal diffusion in double diffusive fluids (e.g., a two-component fluid or a suspension) [6]. Here, the concentration field is coupled to the temperature field through the Soret effect: a temperature gradient leads to a concentration current which in turn influences the density gradient. For a positive Soret effect, the concentration current enhances the thermally induced density gradient, so that convection sets in for a lower temperature difference than in a comparable simple fluid. For certain colloidal suspensions, where this effect is often called thermophoresis, it may be so

\footnotetext{
*Present address: Physikalische und Theoretische Chemie, Technische Universität Braunschweig, Hans-Sommer-Strasse 10, 38106 Braunschweig, Germany.

${ }^{\dagger}$ Present address: Max Planck Institute for Dynamics and SelfOrganization, Am Fassberg 17, 37077 Göttingen, Germany.
}

strong that the density gradient is established predominantly by the Soret effect, so that the resulting convection is almost purely Soret driven. While the time scale of the thermally driven case is given by the relatively small thermal diffusion time, the time scale of Soret-driven experiments is governed by the much larger mass diffusion time. Therefore, even at small laboratory scale experiments, transient effects can last for days or even weeks.

In the present article we investigate the transient convection onset in a colloidal suspension of thermosensitive nanoparticles. Due to the the extraordinarily strong thermophoretic effect of the colloids, the convection in this fluid can be considered as purely Soret driven. In Sec. II, we describe our experimental setup. Our observations are presented in Sec. III and evaluated in Sec. IV. Here, the concentration profile is reconstructed from the shadowgraph observations. Special attention is dedicated to the onset latency time, i.e., the time from switching on the experiment until the onset of convective motion. These latency times and their scaling behaviour are discussed and compared to different models and also to other experiments in Sec. V. We conclude the paper with a brief summary in Sec. VI. In the Appendix, a short outline of one of the boundary layer models leading to transient convection is given.

\section{EXPERIMENTAL SETUP}

The experimental setup and the properties of the working fluid are similar to those presented by Winkel et al. [7]. The fluid is contained in a horizontally aligned, narrow, parallel channel with length $l=70 \mathrm{~mm}$, width $b=1.6 \mathrm{~mm}$, and height $d=3.0 \mathrm{~mm}$, as sketched in Fig. 1. The upper and lower boundaries consist of copper, while the front and back sides are covered by glass plates. A temperature gradient can be applied to the cell by electrically heating the lower copper plate to a temperature $T_{0}+\Delta T$, while the upper plate is held at a fixed temperature $T_{0}$ by a water circulation thermostat. If no heating power is applied, a slightly negative temperature difference of $\Delta T \approx-0.05 \mathrm{~K}$ is established, which is due to the weak thermal coupling of the lower plate to the slightly cooler environment.

The cell is illuminated from the $y$ direction and imaged with a computer-controlled camera from the opposite side, as shown in Fig. 2. To track the convective motion of the fluid, 


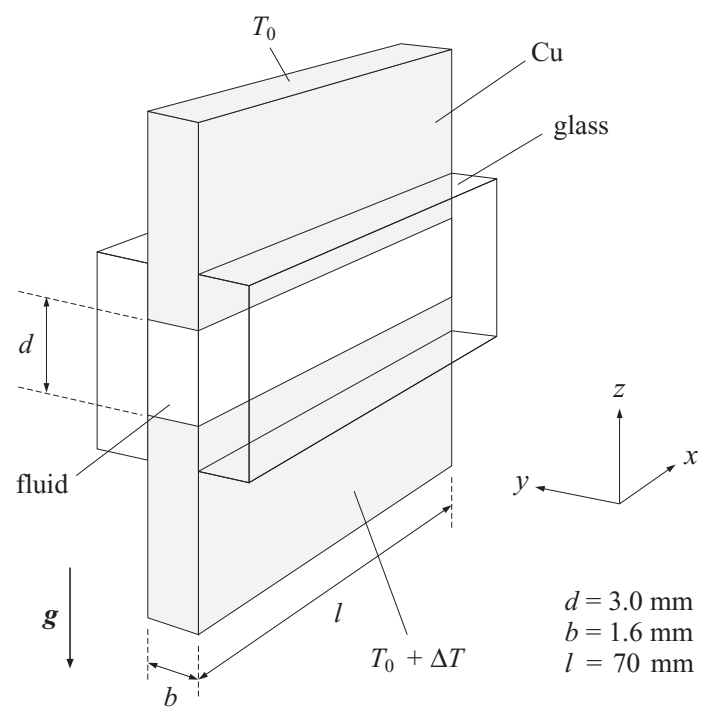

FIG. 1. Schematic representation of the convection cell.

fluorescent polystyrene tracer particles ${ }^{1}$ with a diameter of $2 \mu \mathrm{m}$ and a density comparable to that of the fluid are added. The concentration of tracer particles has a number density of approximately $1900 / \mathrm{mm}^{3}$, corresponding to a mass fraction of approximately $8 \mathrm{ppm}$ of polystyrene in water. Although with this setup we can only measure the $x$ - $z$ position of the particles, a movement in the $y$ direction can be detected qualitatively from the change of the diffraction patterns produced by movements perpendicular to the focus plane.

When the camera is slightly defocused from the fluid layer, shadowgraph images can be observed due to the dependence of the refractive index of the fluid on the temperature and on the colloid concentration [8-10]. For all shadowgraph images presented in this work, the camera was focused to a virtual focal plane as shown in Fig. 2(b). Therefore the fluid that is represented by the darker regions in the shadowgraph images is denser (i.e., has a lower temperature or a higher particle concentration) than the fluid represented by the brighter regions.

\footnotetext{
${ }^{1}$ Sigma-Aldrich L1153.
}
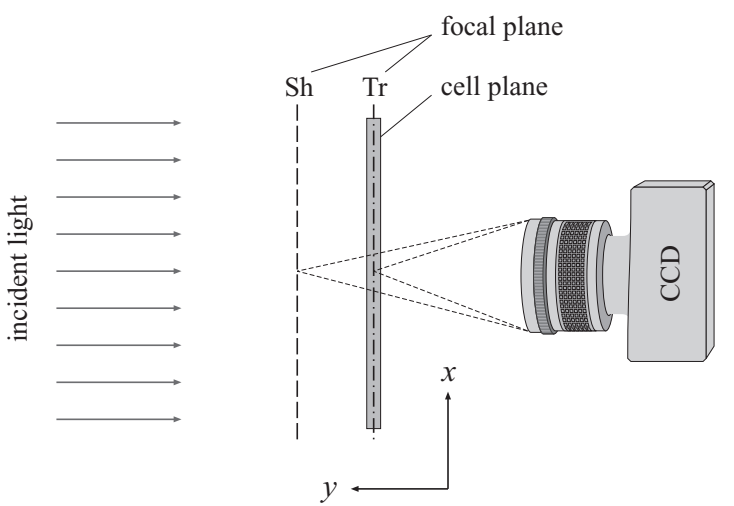

FIG. 2. Sketch of the optical setup. Focal planes for shadowgraphy (Sh - - -) and particle tracking $(\operatorname{Tr}-\cdot-\cdot-)$, respectively.
As working fluids, we use aqueous suspensions of thermosensitive core-shell nanoparticles. ${ }^{2}$ The particles consist of an inner polystyrene core with an attached shell of a poly( $N$-isopropylacrylamide) (PNIPA) gel network [11]. The solubility of the PNIPA shell in water strongly depends on the temperature, leading to a swelling of the gel shell and an increase of the viscosity of the fluid with decreasing temperature [12-14]. The solubility behavior of the PNIPA shell also causes a comparatively strong positive Soret effect of the colloids [15-17]. For our experiments, we use two similar samples of nanoparticles with the internal names KS15 and KS18 that originate from different synthesis runs and differ slightly in the diameter of the core and in the size of the gel shell (see Table I). By varying the colloid concentration in the range from $2 \%$ to $12 \%$, we are able to cover a broad range of viscosities. The most important material properties of the working fluids are listed in Table I.

The large value of the separation ratio $\Psi=\frac{\beta \Delta c}{\alpha \Delta T}$, which measures the ratio of the density gradients caused by mass transport due to the Soret effect and by thermal expansion, shows that in our system, thermodiffusion is indeed the dominant instability mechanism for convection $[6,18]$. We therefore consider the convective instability as purely Soret driven. The appropriate control parameter in this case is the solutal Rayleigh number

$$
R_{\mathrm{s}}=\frac{\beta g d^{3}}{D v} \Delta c,
$$

with

$$
\Delta c=-S_{\mathrm{T}} c(1-c) \Delta T
$$

being the overall final stationary concentration difference over the cell height $d$ caused by the Soret effect when applying a temperature difference $\Delta T$. It is useful to retain the definition for $R_{\mathrm{S}}$ even in cases where the system starts to convect before a stationary state can be established. In the above definitions, $\beta=-\frac{1}{\rho} \frac{\partial \rho}{\partial c}$ is the solutal expansion coefficient, $\alpha=-\frac{1}{\rho} \frac{\partial \rho}{\partial T}$ is the thermal expansion coefficient, $g$ is the acceleration due to gravity, $D$ is the mass diffusivity, $v$ is the kinematic viscosity, and $S_{\mathrm{T}}$ is the Soret coefficient. The respective values for our working fluids are given in Table I.

Under stationary conditions, convection sets in if $R_{\mathrm{S}}$ exceeds the critical value $R_{\mathrm{s}, \mathrm{c}}=720$ [19,20]. The temperature dependency of the solutal Rayleigh number for our working fluids is plotted in Fig. 3 for a layer height of $d=3 \mathrm{~mm}$. It is dominated by the viscosity of the substance and in the concentration range considered here, $R_{\mathrm{S}}$ decreases with increasing concentration. For our setup, the critical temperature difference for the onset of Soret-driven convection according to Eq. (1) is below $0.2 \mathrm{mK}$, which is well beyond our measurement accuracy. To prevent the development of Soret-driven convection prior to switch-on, for several days we keep our experiments at a slightly but definite negative temperature difference of $\Delta T=-0.05 \mathrm{~K}$ before switching. The lowest experimentally achievable positive temperature

\footnotetext{
${ }^{2}$ The thermosensitive colloidal suspensions used in this study where synthesized by Miriam Siebenbürger and Matthias Ballauff.
} 
TABLE I. Material properties of the working fluids at $25^{\circ} \mathrm{C}$. The errors given represent the worst-case measurement uncertainties. All values have been rounded to at least two digits. Reference values for water [21,22] are given where applicable.

\begin{tabular}{|c|c|c|c|c|c|c|c|c|c|}
\hline \multicolumn{3}{|l|}{ Material } & \multirow{2}{*}{$\frac{\mathrm{KS} 18}{11.9(1)}$} & \multirow{2}{*}{$\frac{\mathrm{KS} 15}{8.7(2)}$} & \multirow{2}{*}{$\frac{\mathrm{KS} 18}{7.20(5)}$} & \multirow{2}{*}{$\frac{\mathrm{KS} 18}{5.29(3)}$} & \multirow{2}{*}{$\frac{\mathrm{KS} 18}{2.28(1)}$} & \multirow{2}{*}{$\frac{\mathrm{KS} 18}{1.91(1)}$} & \multirow{2}{*}{$\frac{\text { Water }}{-}$} \\
\hline Mass concentration & $c$ & $(\%)$ & & & & & & & \\
\hline Hydrodyn. radius & $R_{h}$ & $(\mathrm{~nm})$ & $70(1)$ & $77(1)$ & $70(1)$ & $70(1)$ & $70(1)$ & $70(1)$ & - \\
\hline Volume fraction & $\Phi$ & & $0.48(5)$ & $0.32(5)$ & $0.29(5)$ & $0.21(2)$ & $0.093(10)$ & $0.077(10)$ & - \\
\hline Density & $\rho$ & $\left(10^{3} \mathrm{~kg} \mathrm{~m}^{-3}\right)$ & $1.008(1)$ & $1.0053(1)$ & $1.0042(1)$ & $1.0023(1)$ & $0.9993(1)$ & $0.9989(1)$ & $0.997047(1)$ \\
\hline Thermal expansion & $\alpha$ & $\left(10^{-4} \mathrm{~K}^{-1}\right)$ & $3.1(1)$ & $2.9(1)$ & $2.9(1)$ & $2.8(1)$ & $2.7(1)$ & $2.7(1)$ & $2.572922(3)$ \\
\hline Solutal expansion & $\beta$ & $\left(10^{-2}\right)$ & $-9.84(4)$ & $-9.46(4)$ & $-9.89(4)$ & $-9.90(4)$ & $-9.93(4)$ & $-9.94(4)$ & - \\
\hline Dynamic viscosity & $\eta$ & $\left(10^{-3} \mathrm{~Pa} \mathrm{~s}\right)$ & $17(6)$ & $6.6(6)$ & $3.4(5)$ & $2.2(3)$ & $1.29(15)$ & $1.21(10)$ & $0.8900(15)$ \\
\hline Kinematic viscosity & $v$ & $\left(10^{-6} \mathrm{~m}^{2} \mathrm{~s}^{-1}\right.$ & $17(6)$ & $6.5(5)$ & $3.4(5)$ & $2.2(3)$ & $1.29(15)$ & $1.21(10)$ & $0.893(1)$ \\
\hline Thermal diffusivity & $\kappa$ & $\left(10^{-7} \mathrm{~m}^{2} \mathrm{~s}^{-1}\right)$ & $1.33(10)$ & $1.36(5)$ & $1.38(5)$ & $1.40(5)$ & $1.43(4)$ & $1.43(4)$ & $1.46(3)$ \\
\hline Mass diffusivity & $D$ & $\left(10^{-12} \mathrm{~m}^{2} \mathrm{~s}^{-1}\right)$ & $8.6(2)$ & $6.8(4)$ & $7.1(5)$ & $6.7(5)$ & $5.9(6)$ & $5.8(7)$ & - \\
\hline Soret coefficient & $S_{\mathrm{T}}$ & $\left(\mathrm{K}^{-1}\right)$ & $0.27(2)$ & $0.35(3)$ & $0.54(3)$ & $0.54(3)$ & $0.63(6)$ & $0.65(6)$ & - \\
\hline Refractive index & $n$ & & $1.356(2)$ & $1.3507(2)$ & $1.347(1)$ & $1.343(1)$ & $1.3364(4)$ & $1.3356(4)$ & $1.33162(2)$ \\
\hline$(632.8 \mathrm{~nm})$ & $\partial n / \partial T$ & $\left(10^{-4} \mathrm{~K}^{-1}\right)$ & $-1.7(4)$ & $-1.3(1)$ & $-1.4(3)$ & $-1.3(2)$ & $-1.2(1)$ & $-1.2(1)$ & -1.055090 \\
\hline & $\partial n / \partial c$ & & $0.21(1)$ & $0.23(1)$ & $0.21(1)$ & $0.21(1)$ & $0.21(1)$ & $0.21(1)$ & - \\
\hline Prandtl number & $\operatorname{Pr}=v /$ & & $127(50)$ & $48(4)$ & $25(4)$ & $16(2)$ & $9.0(10)$ & $8.5(7)$ & $6.13(12)$ \\
\hline Schmidt number & $\mathrm{Sc}=v$ & $\left(10^{5}\right)$ & $2.0(7)$ & $9.5(9)$ & $4.8(8)$ & $3.4(5)$ & $2.2(3)$ & $2.1(3)$ & - \\
\hline Lewis number & $\mathrm{Le}=D$ & $\left(10^{-5}\right)$ & $6.5(5)$ & $5.0(3)$ & $5.2(4)$ & $4.8(4)$ & $4.1(4)$ & $4.1(5)$ & - \\
\hline Separation ratio & $\Psi=-$ & ${ }_{\mathrm{T}} c(1-c)$ & $8.9(7)$ & $8.8(8)$ & $12(8)$ & $9.6(6)$ & $5.2(5)$ & $4.5(4)$ & - \\
\hline
\end{tabular}

difference in our experiments is $\Delta T=0.05$. A comparison of the resulting solutal Rayleigh numbers $R_{\mathrm{s}}>10^{5}$ with the critical value $R_{\mathrm{s}, \mathrm{c}}=720$ for the onset of convection shows that our experiments always run in a strongly overcritical regime even for the lowest possible temperature differences. On the other hand, our highest applied temperature difference of $\Delta T \approx 6 \mathrm{~K}$ is still well below the critical temperature difference for purely thermally driven convection, which in our setup is $\Delta T_{\mathrm{c}}^{\mathrm{w}}=(9.82 \pm 0.01) \mathrm{K}$ for water.

\section{EXPERIMENTAL OBSERVATIONS}

In the experiments discussed in this paper, the temperature difference is suddenly switched from a slightly negative

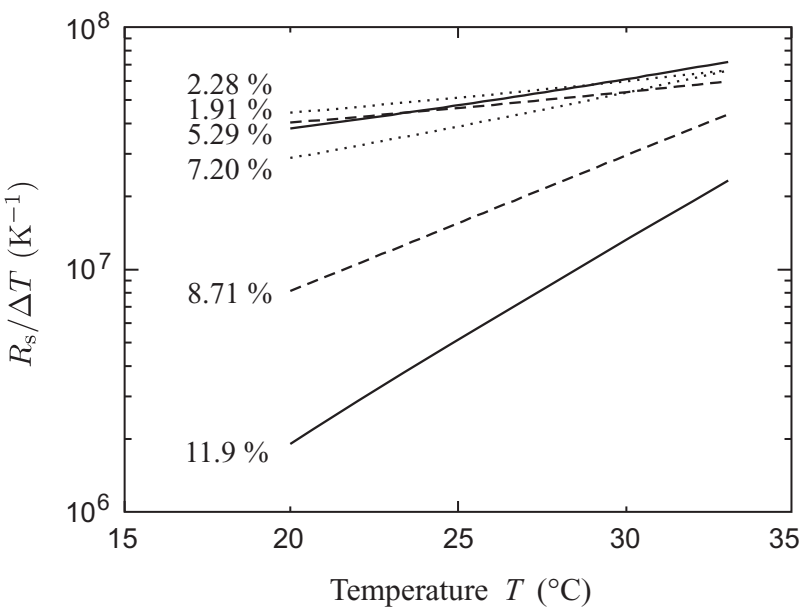

FIG. 3. Temperature dependence of the relevant material parameter combination $-\frac{\beta g d^{3}}{D v} S_{\mathrm{T}} c(1-c)=R_{\mathrm{S}} / \Delta T$ for the working fluids $\mathrm{KS} 15(8.71 \%)$ and $\mathrm{KS} 18(1.91 \%, 2.28 \%, 5.29 \%, 7.20 \%, 11.9 \%)$ in a cell of height $d=3 \mathrm{~mm}$. value of $\Delta T \approx-0.05 \mathrm{~K}$ to different positive values. Figure 4 shows shadowgraph images taken after switching to a solutal Rayleigh number of $R_{\mathrm{s}}=1.3 \times 10^{8}$. The corresponding video recording is available online [23]. After some latency time $t_{\text {lat }}$, a front detaches from the bottom and propagates into the cell [Figs. 4(a)-4(c)]. Some minutes later, the same course of events can be observed starting at the upper boundary, which is not shown here. After a disordered interlude lasting for several hours, a stationary convection pattern establishes

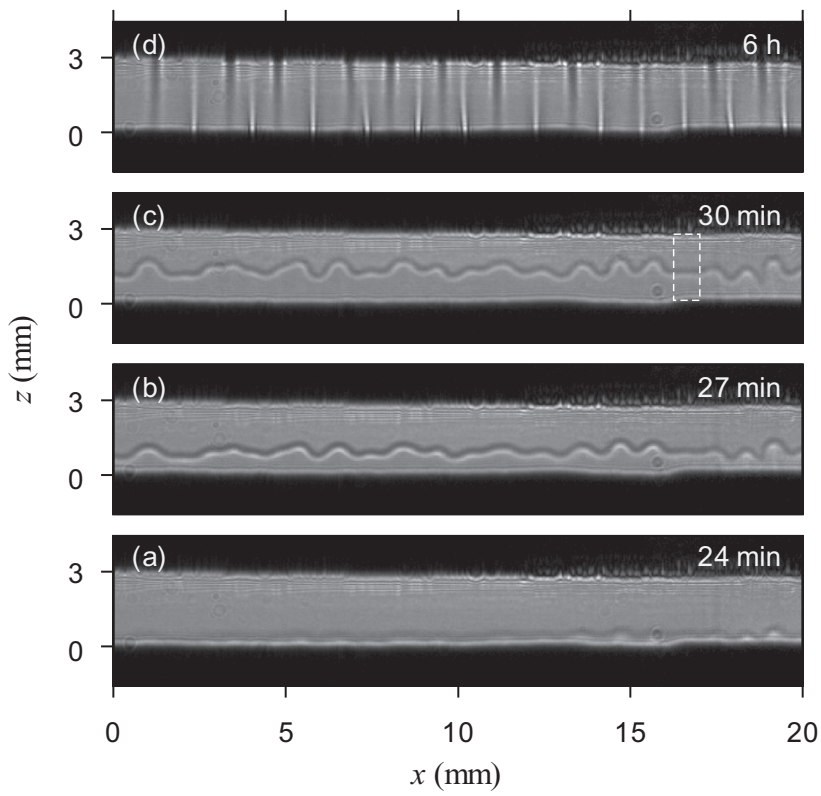

FIG. 4. Shadowgraph images at different times after switching from a subcritical state to a solutal Rayleigh number of $R_{\mathrm{s}}=$ $1.3 \times 10^{8}$. The region marked in image (c) is shown in Fig. 7 in a magnified version. 


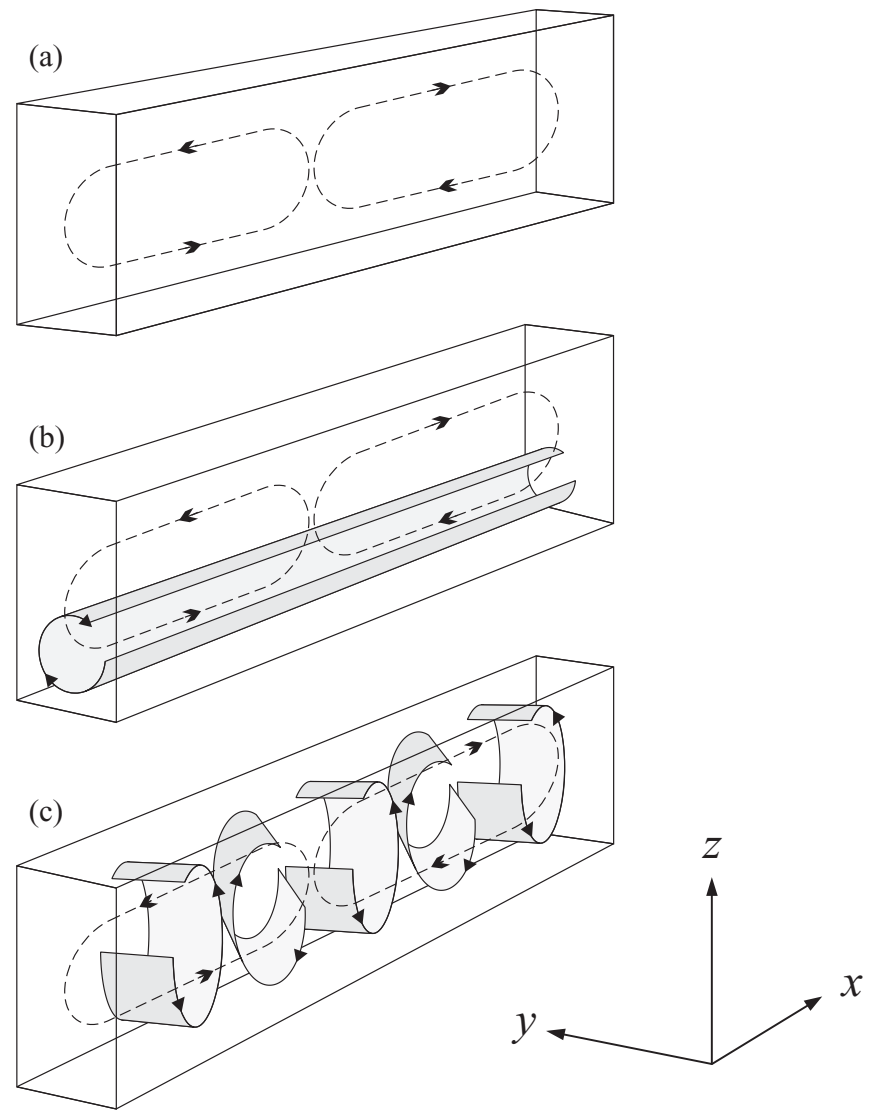

FIG. 5. Schematic representation of the fluid flow at different phases. (a) Primary flow before the convection onset, which is always present (see also b,c). (b) Secondary flow at the convection onset. (c) Splitting of the secondary flow into several rolls.

[Fig. 4(d)]. Note that the rising front [Figs. 4(b) and 4(c)] shows a spatial modulation with a wavelength of the same order as the final stationary pattern [Fig. 4(d)]. This modulation becomes more pronounced with increasing temperature differences. For Rayleigh numbers well above $10^{8}$, we observe that the up- and downrising fronts completely split into discrete plumes (not shown here).

Particle tracking observations reveal some more characteristics of the fluid motion which are sketched schematically in Fig. 5. Under all conditions (i.e., subcritical and supercritical), we observe a very slow, large-scale fluid motion in the form of one or two vortex rolls spanning over the entire cell [Fig. 5(a)]. This flow has a maximum velocity of about $1 \mathrm{~mm} / \mathrm{h}$ and is probably caused by a slightly inhomogeneous temperature distribution or an imperfect horizontal alignment of the cell, which leads to convection at arbitrarily small temperature differences $[24,25]$. It should be noted that the Soret-driven convection has a critical wave number of $k_{\mathrm{c}}=0[6,19,20]$. For slightly overcritical solutal Rayleigh numbers, Soret-driven convection should therefore manifest itself in the form of one or two rolls, as observed here. However, as described in Sec. II, we prevent the development of this convection mode by applying a slightly negative temperature difference before switching.

When switching to a supercritical Rayleigh number, we observe the onset of a distinct convective motion, even before

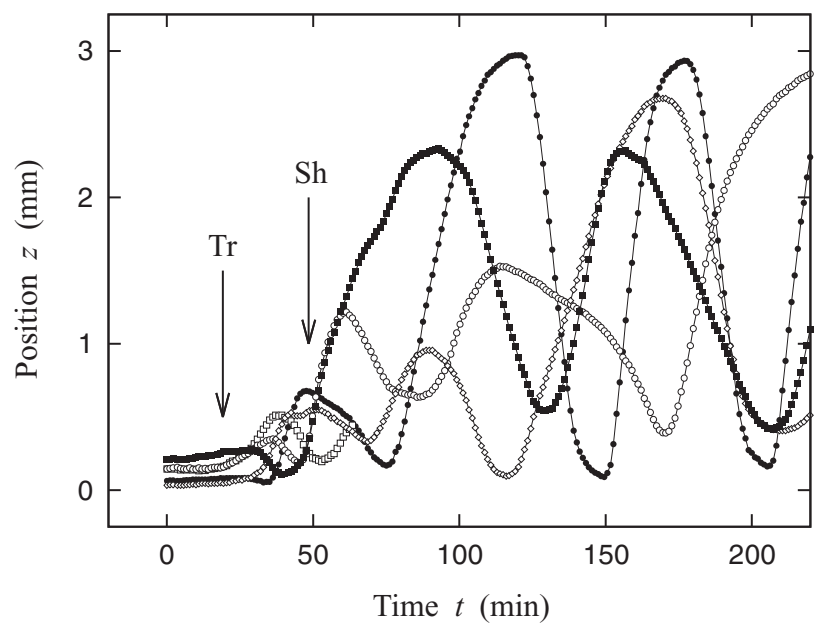

FIG. 6. Temporal development of the vertical position of four tracked particles after the solutal Rayleigh number has been switched from a subcritical value to $R_{s}=1.4 \times 10^{7}$ at time $t=0$. The arrows mark the onset latency times evaluated from the particle tracking observations ( $\mathrm{Tr}$ ) and from the shadowgraph evaluation (Sh), respectively (see Sec. IV B)

the appearance of the rising shadowgraph front. This motion always starts in the form of a single vortex roll at the lower boundary, with its axis being aligned in the $x$ direction (i.e., parallel to the primary flow direction), and grows upwards with time, propagating the fluid motion into the cell [Fig. 5(b)]. Some time after the onset at the lower boundary, a second, parallel convection roll emerges at the upper boundary. After meeting in the middle, the two opposingly growing rolls develop into a single roll, which stretches over the full height of the cell. This resulting roll eventually splits into a stripe pattern of convection rolls which reorient towards the $y$ direction [Fig. 5(c)]. An exemplary video of a particle tracking recording is available online [23].

The vertical position of some tracked particles, initially located near the lower boundary, are shown in Fig. 6. The arrows mark the onset of a vertical movement seen in the particle tracking experiments $(\mathrm{Tr})$ and the time of the appearance of the front in the shadowgraph observations (Sh), respectively. For more details on the definition of the onset latency times, refer to Sec. IV B. The increasing height of the upper turnover points corresponds to the growing extension of the convection roll into the cell. After approximately $100 \mathrm{~min}$, the convection roll has occupied the complete height of the cell.

In the final stationary configuration, after splitting into a stripe pattern the rolls are aligned almost parallel to the $y$ direction. Visual inspection, however, reveals a small but distinct velocity component of the tracer particles in the $y$ direction which is most pronounced towards the top and bottom of the cell. This indicates a residual angle of the roll to the $y$ axis and is in agreement with theoretical considerations by Davies-Jones [26], who argued that convection rolls can never be aligned exactly perpendicular to realistic boundaries. We will not discuss this and other features of the stationary convection patterns further but will rather focus the following discussion on the transient behavior at the convection onset. 

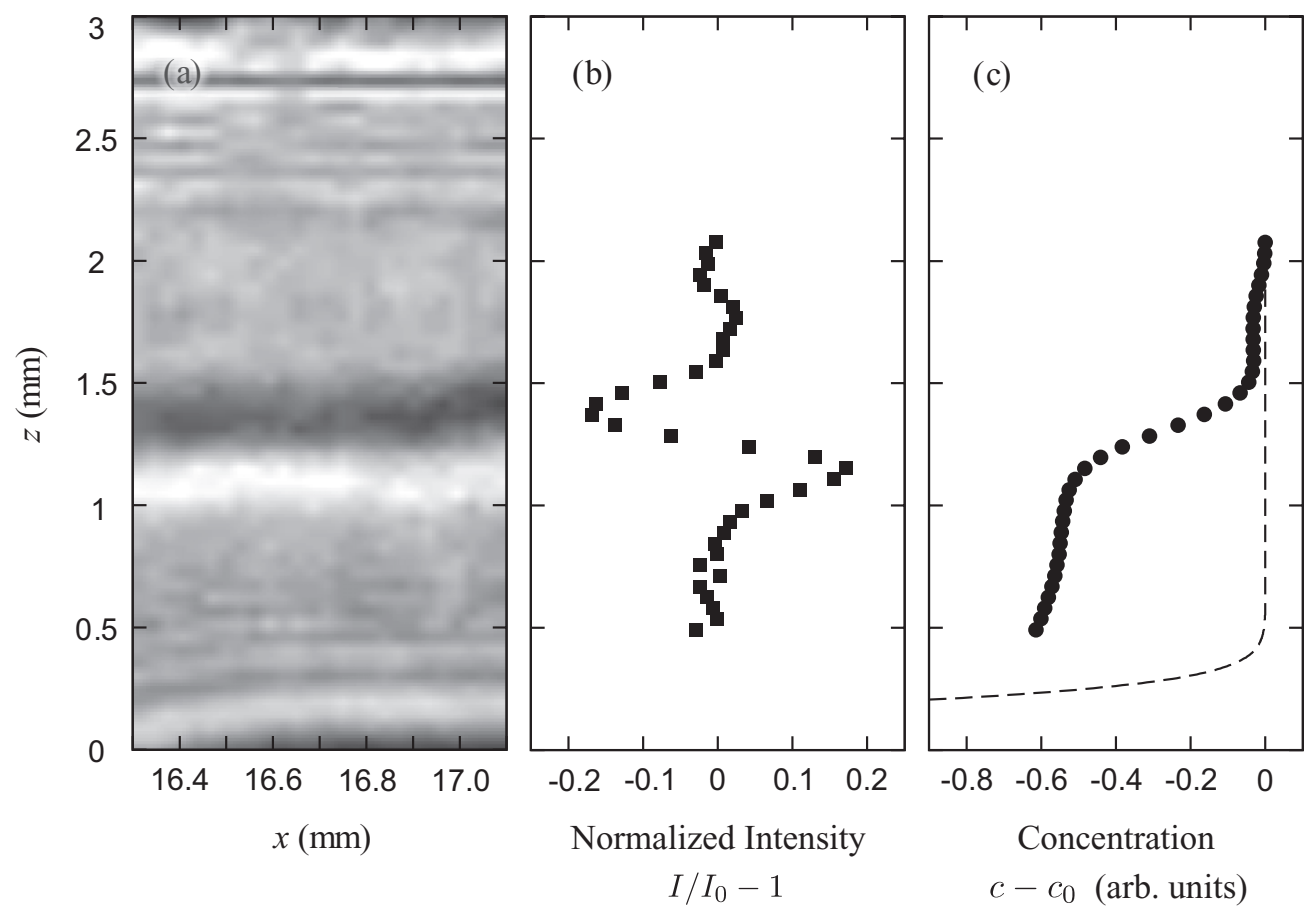

FIG. 7. Reconstruction of the concentration profile. (a) Shadowgraph image [magnification from Fig. 4(c)]. (b) (ם) Normalized shadowgraph intensity, averaged over $x \in[16.3 \ldots 17.1] \mathrm{mm}$. (c) (•) Concentration profile reconstructed from the shadowgraph intensity by double integration according to Eq. (4). Only the range $z=0.5 \ldots 2.1 \mathrm{~mm}$ has been evaluated. (- - ) Theoretical concentration profile corresponding to a purely diffusive boundary layer according to Eq. (A8).

\section{EVALUATION AND INTERPRETATION OF THE OBSERVATIONS}

\section{A. Reconstruction of the concentration profile from the shadowgraph images}

Figure 7(a) shows a magnification of the part of the shadowgraph image which has been marked in Fig. 4(c) and which will now be evaluated in detail. The rising front is represented by the strong intensity modulation (dark and bright region) near $z \approx 1.3 \mathrm{~mm}$. The intensity oscillations near the cell boundaries at the top and bottom are interference patterns of the light reflected by the metallic horizontal surfaces and are not of interest here.

If the variation of the refractive index over the fluid layer is small, the normalized intensity of the shadowgraph images can be approximated up to linear order to be proportional to the curvature of the refractive index $n$ [8-10]:

$$
\frac{I-I_{0}}{I_{0}} \propto \frac{d^{2} n}{d z^{2}} .
$$

Here, $I_{0}$ is the intensity field in the subcritical state ("zero intensity"). In our fluids and in the temperature regime of interest, the refractive index depends approximately linearly on both the temperature and the colloid concentration: $n(c, T)-n\left(c_{0}, T_{0}\right)=\frac{\partial n}{\partial c}\left(c-c_{0}\right)+\frac{\partial n}{\partial T}\left(T-T_{0}\right)$. Higher-order dependencies of $n$ on $c$ and $T$ contribute less than 5\%. Values for $\frac{\partial n}{\partial c}$ and $\frac{\partial n}{\partial T}$ are given in Table I. Due to the fact that in our system thermal diffusion is much faster than mass diffusion, the temperature field has already relaxed to its linear stationary state for the time scales considered here. Therefore the temperature field is not visible in the shadowgraph images, thus restricting the intensity dependency to the concentration field only:

$$
\frac{I-I_{0}}{I_{0}} \propto \frac{\partial n}{\partial c} \frac{d^{2} c}{d z^{2}}+\frac{\partial n}{\partial T} \underbrace{\frac{d^{2} T}{d z^{2}}}_{=0} .
$$

The normalized shadowgraph intensity, averaged over the $x$ range displayed in Fig. 7(a), is given in Fig. 7(b). The zero-intensity $I_{0}$ has been taken from images recorded at the beginning of the experiment in the subcritical state. The regions with the intensity oscillations near the cell boundaries have not been included in the diagram in order to highlight the region of the rising front. It is in principal possible to reconstruct the concentration profile over the cell (except for an unknown linear term) by using Eq. (4) and twice integrating the intensity data shown in Fig. 7(b). However, due to the uncertainties introduced by a double integration of biases in the shadowgraph images and by the two unknown integration constants, quantitative results obtained by this method may be of questionable significance. We will therefore restrict the discussion to the qualitative form of the concentration profile.

Figure 7(c) shows the result of this procedure. The intensity data given in Fig. 7(b) has been integrated twice in the negative $z$ direction starting from $z=2.1 \mathrm{~mm}$, where the concentration is assumed to equal the initial value $c_{0}$ with the slope $\frac{d c}{d z}=0$. The distinct zero-crossing of the image intensity shown in Fig. 7(b) corresponds to an inflection point in the concentration profile, apparent in Fig. 7(c). For comparison, the purely diffusive concentration profile that would be established in the cell at the time of the image recording when no convection would be present [see Eq. (A8)] 


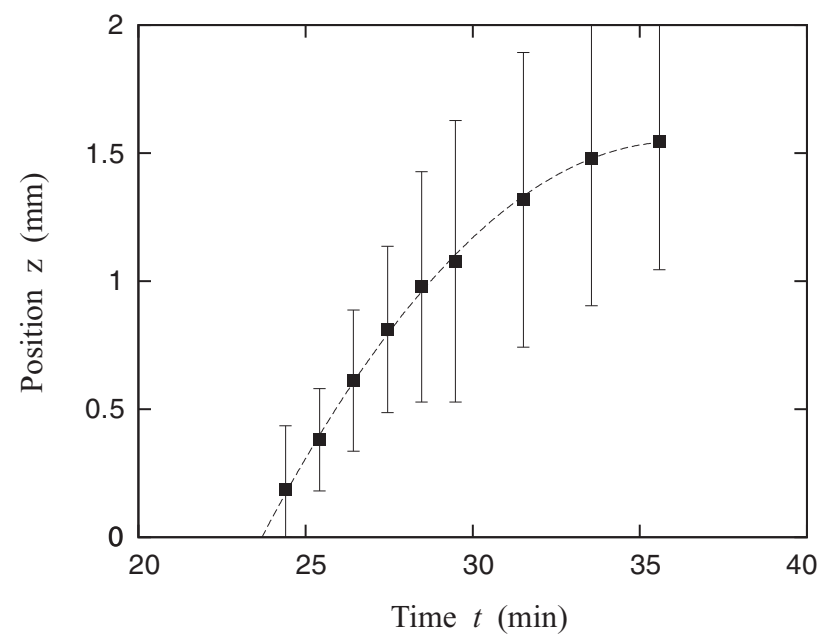

FIG. 8. (ם) Average position of the rising convection front after switching from a subcritical value to a solutal Rayleigh number of $R_{s}=1.3 \times 10^{8}$. The vertical bars are not error bars but rather represent the peak extensions of the modulations. (- - ) Fitted secondorder polynomial $z=a\left(t-t_{\text {lat }}\right)+b\left(t-t_{\text {lat }}\right)^{2}$ for the extrapolation of the onset latency time $t_{\text {lat }}$.

is also given, with the amplitude $\Delta c$ in Eq. (A8) being scaled to match the integrals of the two concentration fields. Obviously, the purely diffusive profile cannot account for the observed shadowgraph images, since it is growing significantly too slow and also shows the wrong curvature. We therefore interpret the observations such that the diffusive concentration boundary layer has been remixed by the uprising roll visible in the particle tracking observations described earlier and sketched in Fig. 5(b). The rising front in the shadowgraph images shown in Figs. 4(a)-4(c) then corresponds to the upper margin of this uprising roll.

\section{B. Extraction of the onset latency times}

When suddenly switching the temperature difference from a subcritical to a supercritical value, convection does not set in instantaneously, but rather after some latency time $t_{\text {lat }}$. These times will now be extracted for different temperature differences from both the shadowgraph recordings and the particle tracking observations.

From the shadowgraph images, the average position $z$ of the uprising front is tracked as a function of time. The result for a jump to a solutal Rayleigh number of $R_{s}=1.3 \times 10^{8}$ is shown in Fig. 8. Since the lower cell boundary is not very well visible in the recordings, the quadratic function $z(t)=$ $a\left(t-t_{\text {lat }}\right)+b\left(t-t_{\text {lat }}\right)^{2}$ is fitted to the data shown in Fig. 8 in order to extrapolate the latency time $t_{\text {lat }}$.

For the particle tracking experiments, the onset of a distinctly visible vertical movement of the tracer particles is interpreted as the onset latency time. For a quantitative evaluation, we track the vertical position of particles initially located near the lower cell boundary as functions of time. The position of such a particle is then fitted by the piecewise linear function

$$
z= \begin{cases}z_{\mathrm{c}}+m_{\mathrm{f}}\left(t-t_{\mathrm{c}}\right), & t \geqslant t_{\mathrm{c}} \\ z_{\mathrm{c}}+m_{\mathrm{c}}\left(t-t_{\mathrm{c}}\right), & t<t_{\mathrm{c}}\end{cases}
$$

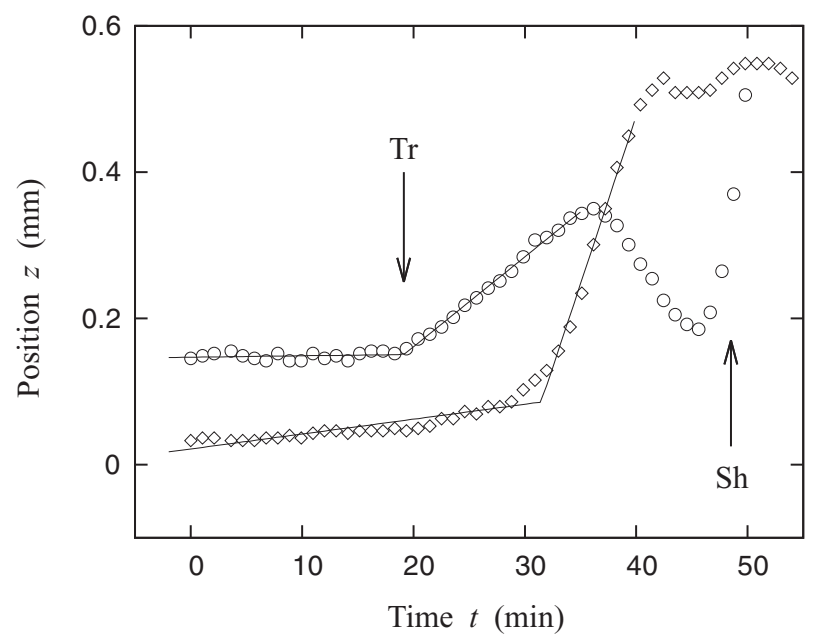

FIG. 9. Vertical position of two tracked particles shortly after a switch from a subcritical value to a solutal Rayleigh number of $R_{s}=1.4 \times 10^{7}$ at $t=0$. (This figure is a detail of Fig. 6.) To extract the onset latency time, the data prior to the first maxima is fitted by the piecewise linear function given by Eq. (5) (-). The arrows mark the resulting onset latency time ( $\mathrm{Tr}$ ) and the corresponding value according to the shadowgraph evaluation (Sh).

where only the range prior to the first maximum is considered. The minimal $t_{\mathrm{c}}$ obtained from all particles considered is taken as the onset latency time $t_{\text {lat }}$. An example of two particles tracked and evaluated in this way is given in Fig. 9.

The onset latency times obtained from both sets of experiments have been normalized with the mass diffusion time

$$
\tau_{\mathrm{D}}=d^{2} / D
$$

and are plotted in Fig. 10 against the solutal Rayleigh number $R_{\mathrm{s}}$. For the calculation of the material-parameter-dependent part of $R_{\mathrm{S}}$, the temperature at the cell bottom has been used, since this is the position where the convective motion sets in. Obviously, the latency times extracted via particle tracking are systematically lower than those obtained from the shadowgraph images by a factor of about 2 . Within the accuracy of the measurements, the latency times observed for the shadowgraph experiments follow the scaling relation

$$
\frac{t_{\text {lat }}}{\tau_{\mathrm{D}}}=(12 \pm 3) R_{\mathrm{s}}^{-0.51 \pm 0.01}
$$

while for the particle tracking evaluation we find

$$
\frac{t_{\text {lat }}}{\tau_{\mathrm{D}}}=(4 \pm 1) R_{\mathrm{s}}^{-0.49 \pm 0.03} .
$$

\section{DISCUSSION: SCALING BEHAVIOR OF THE ONSET LATENCY TIMES}

\section{A. Comparison with theory}

An instructive theoretical estimate for the onset latency time has been presented by Howard [27] for thermally driven convection at high Rayleigh numbers. We will compare our data to the results of this method applied to the Soret-driven case. Details can be found in Messlinger et al. [28] and in the Appendix. 


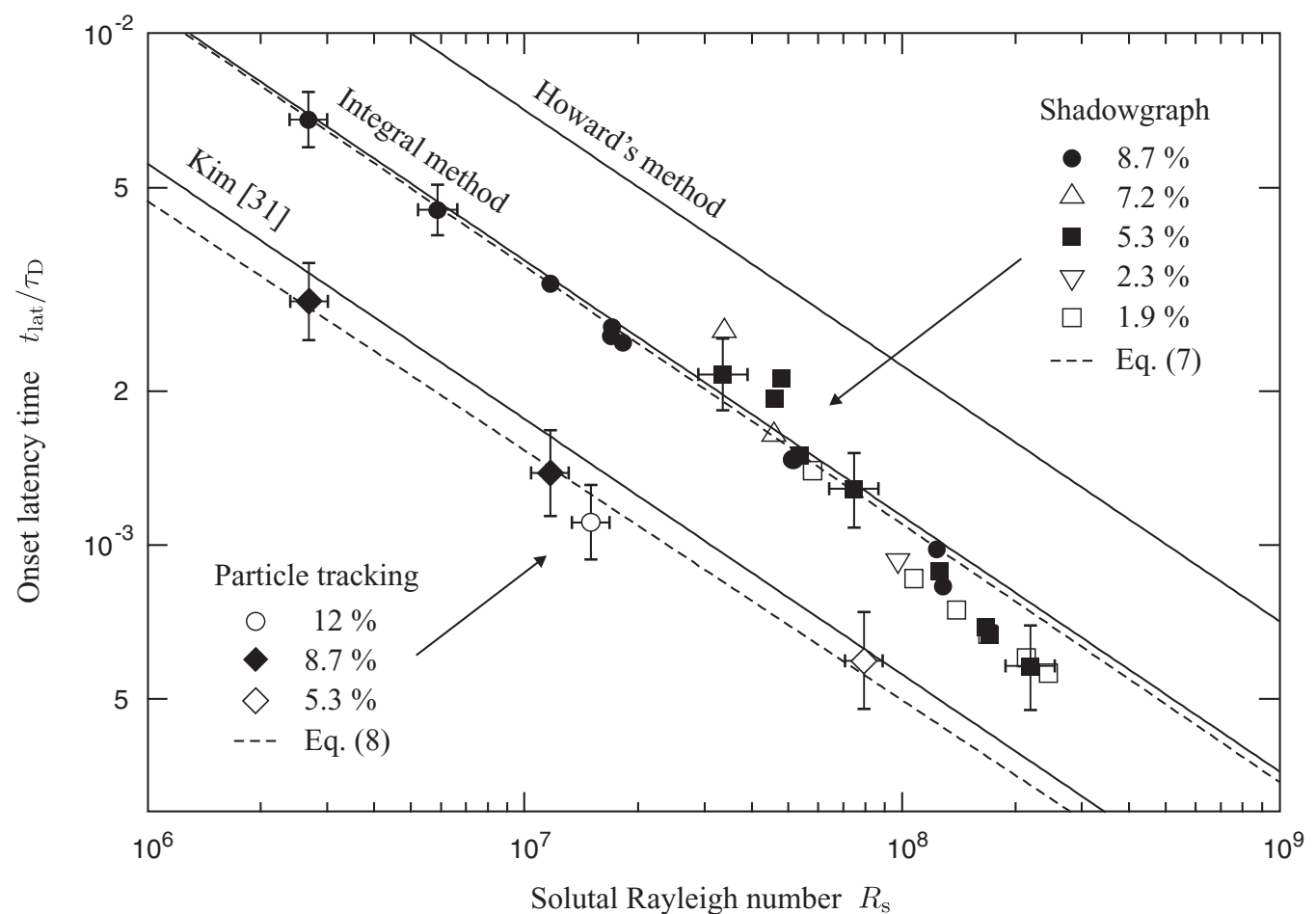

FIG. 10. Onset latency times observed with particle tracking and with shadowgraph imaging, using the substances KS15 (8.71\%) and KS18 $(1.91 \%, 2.28 \%, 5.29 \%, 7.20 \%, 11.9 \%)$. In order to improve readability, error bars are only given for exemplary data points. (- - -) Fitted exponential scaling laws given by Eqs. (7) and (8). (-) Theoretical models according to Howard's method [Eq. (11)], the integral method [Eq. (12)], and Kim [31] [Eq. (13)].

After suddenly switching the bottom plate to a higher temperature than the initially assumed homogeneous fluid layer, mass is transported away from the boundary due to the Soret effect. The concentration profile established by this mass flux can be determined analytically [Eq. (A7)] and is shown for different times after the temperature switch in Fig. 11. Note that the impermeable top and bottom walls enforce the total concentration current at the boundaries to vanish, leading to a fixed concentration gradient near the boundaries already

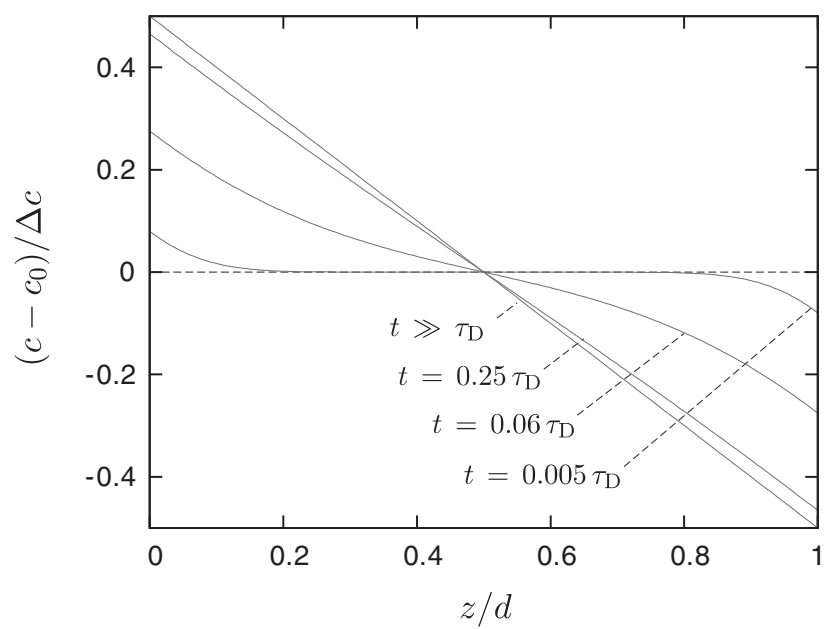

FIG. 11. Evolution of the diffusive concentration profile with Neumann boundary conditions according to Eq. (A7). shortly after the switch-on that counterbalances the Soret current with an opposing diffusion current. Therefore near the boundary the profile may be a approximated by a linear profile with the slope given by the gradient at the cell boundary, as shown in Fig. 12. The height $\delta$ of this concentration boundary layer is then defined by the zero-crossing of the concentration profile (Fig. 12) and increases with the square root of time, as does the peak concentration at the bottom plate (see Fig. 11). It is assumed that the linearized layer becomes unstable against

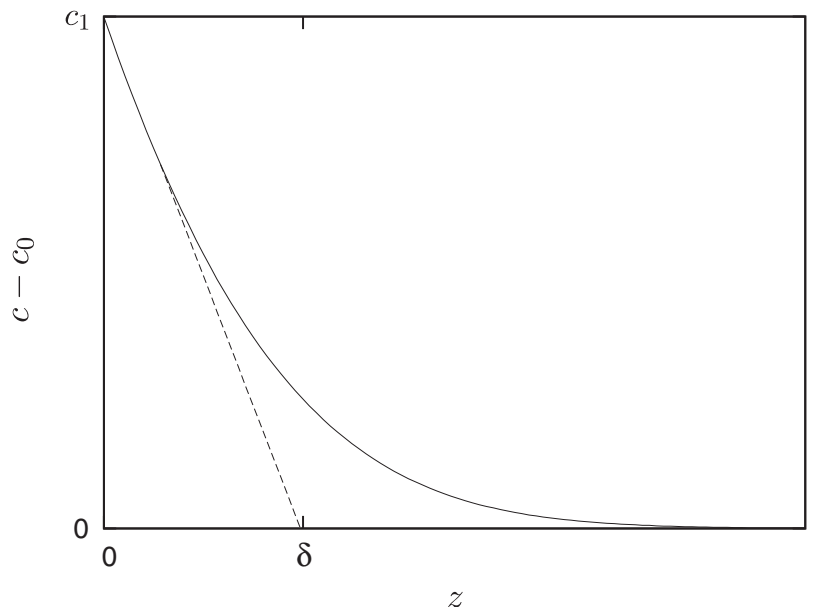

FIG. 12. Concentration profile near the boundary for $t \ll \tau_{\mathrm{D}}$. (-) Temperature profile according to Eq. (A8). (- - ) Approximated linear profile of height $\delta$ according to Eq. (A10). 
convection once the "local" solutal Rayleigh number

$$
r_{\mathrm{s}}=\frac{\beta g \delta^{3}}{D v} \Delta c
$$

corresponding to Eq. (1) but calculated over the boundary layer height $\delta$ only, exceeds the threshold criterium for a stationary profile, i.e., for $r_{\mathrm{s}}>R_{\mathrm{s}, \mathrm{c}}$. This happens at a critical boundary layer height $\delta^{*}$,

$$
\frac{\delta^{*}}{d}=\left(\frac{R_{\mathrm{s}}}{R_{\mathrm{s}, \mathrm{c}}}\right)^{-1 / 4} \approx 5.34 R_{\mathrm{s}}^{-1 / 4},
$$

which is reached after the latency time $t_{\text {lat }}$,

$$
\frac{t_{\text {lat }}}{\tau_{\mathrm{D}}}=\frac{\pi}{4}\left(\frac{R_{\mathrm{s}}}{R_{\mathrm{s}, \mathrm{c}}}\right)^{-1 / 2} \approx 22.4 R_{\mathrm{s}}^{-1 / 2},
$$

after the switch-on. In this formulation, the explicit dependencies on the material parameters of the fluid are hidden in the global Rayleigh number $R_{\mathrm{S}}$, which is also a convenient macroscopic control parameter for the experiment. For the explicit prefactors given on the right-hand sides of Eqs. (10) and (11), a critical Rayleigh number of $R_{\mathrm{s}, \mathrm{c}}=816.74$ has been assumed $[19,20]$. This corresponds to a mechanically rigid lower and stress-free upper boundary, in order to account for the possibility of lateral fluid motion at the upper side of the concentration boundary layer, which is typically well inside the fluid layer and not close to the rigid upper wall. Since $t_{\text {lat }}$ only depends on the square root of $R_{\mathrm{s}, \mathrm{c}}$, however, the actual choice of the value for $R_{\mathrm{S}, \mathrm{c}}$ does not change the magnitude of $t_{\text {lat }}$ significantly.

Alternatively, as proposed by Shliomis and Souhar [29], the height of the concentration boundary layer may be defined by requiring the integral over the concentration profile to be conserved. This leads to a reduced prefactor in the scaling relation for the onset latency time:

$$
\frac{t_{\text {lat }}}{\tau_{\mathrm{D}}}=\frac{1}{\sqrt{2 \pi}}\left(\frac{R_{\mathrm{s}}}{R_{\mathrm{s}, \mathrm{c}}}\right)^{-1 / 2} \approx 11.4 R_{\mathrm{s}}^{-1 / 2} .
$$

Note that for both methods, the stability criteria applied here are valid only for horizontally infinitely extended fluid layers, while for narrow channels, other scaling relations apply [28]. Although the experiments discussed here are run in a narrow channel rather than in a bulk fluid layer, the critical layer heights at the convection onset according to Eq. (10) are significantly smaller than the cell thickness: $\delta^{*}<0.1 d<$ $0.2 b$ for the solutal Rayleigh numbers of $R_{\mathrm{S}}>10^{6}$ applied here. Therefore, at the convection onset, the cell boundaries are still far away compared to the concentration layer height, so that the scaling relation for the bulk geometry is appropriate.

It is well known that the stability analyses of nonlinear temperature profiles yield significantly lower critical Rayleigh numbers than those of the corresponding linear profiles [30]. A stability analysis of the full nonlinear transient profile evolving in a Soret-driven experiment has been presented by Kim [31], who reports a scaling relation for the onset time of

$$
\frac{t_{\text {lat }}}{\tau_{\mathrm{D}}} \approx 5.58 R_{\mathrm{s}}^{-1 / 2} .
$$

The theoretical scaling relations [Eqs. (11)-(13)] derived by the different methods are shown together with the experimental data in Fig. 10. The exponential scaling behavior of the onset latency times measured in our experiments is in good agreement with these models. The absolute values extracted from the shadowgraph recordings coincide very well with the prefactor in Eq. (12), derived by the integral method, while the values obtained from the particle tracking recordings agree with the result of Eq. (13) by Kim [31].

The discrepancy of the onset latency times predicted by the theoretical treatment and observed experimentally by the shadowgraph method is well known. It is often attributed to the fact that after the real latency time, some additional growth period has to pass until the convection flow is sufficiently strong to be experimentally detectable with the shadowgraph method [31,32]. This argument is supported by our observation, where the convection onset detected with particle tracking coincides well with the order of magnitude predicted by the theory, while the shadowgraph evaluation yields systematically larger values.

\section{B. Comparison with other experiments}

Cerbino et al. [33] have investigated Soret-driven convection of a colloidal suspension with a large negative separation ratio in a bulk experiment heated from above at high solutal Rayleigh numbers of $R_{\mathrm{s}}=10^{6} \ldots 10^{9}$. They report the onset latency time to follow the relation $t_{\text {lat }} / \tau_{\mathrm{D}}=25.3 R_{\mathrm{s}}^{-0.52 \pm 0.03}$.

Nielsen and Sabersky [34] observed the onset of thermally driven convection of silicone oils in a bulk geometry heated from below with a constant heat flux over the bottom boundary. Due to this boundary condition, their system can be considered mathematically equivalent to the Soret-driven case, if the solutal Rayleigh number is replaced by the normal Rayleigh number $R$. From their data we extract a scaling for the onset latency times of $t_{\text {lat }} / \tau_{\mathrm{D}}=20 R^{-0.5}$.

In both studies, the convection was observed with the shadowgraph technique, looking from above. Both scaling relations show the same exponential behavior as in our experiments, however, with slightly larger prefactors. Keeping in mind the different geometries and observation techniques of the experiments, the agreement of the onset latency times is amazingly good.

\section{SUMMARY AND CONCLUSION}

We have investigated the onset of transient, Soret-driven convection experimentally by particle tracking and shadowgraph methods. The onset latency times evaluated from both methods obey the theoretically predicted exponential scaling laws. However, particle tracking observations reveal that the convective fluid motion sets in significantly earlier than detectable by the shadowgraph method. The onset latency time extracted from particle tracking observations is most accurately described by the theoretical analysis of Kim [31]. Although our experiments were conducted in a narrow cell, in the range of large solutal Rayleigh numbers investigated here, it is plausible that the convection onset in a bulk fluid layer shows a similar behavior. The respective particle tracking experiments in bulk geometries have yet to be done. 


\section{ACKNOWLEDGMENTS}

We thank Roberto Cerbino, Alberto Vailati, Gora Conley, and Simon Wongsuwarn for stimulating discussions, and Andreas Königer and Werner Köhler for their helpful advice concerning the measurement of refractive indices and Soret coefficients. Special thanks goes to Miriam Siebenbürger and Matthias Ballauff for providing the PNIPA core-shell colloids. Financial support of the Deutsche Forschungsgemeinschaft (DFG) through the Forschergruppe FOR608, Convection in Suspensions project is gratefully acknowledged.

\section{APPENDIX: LATENCY TIME SCALING FOR THE CONVECTION ONSET IN A TRANSIENT DIFFUSIVE BOUNDARY LAYER ESTABLISHED BY THE SORET EFFECT}

We consider a fluid layer of height $d$, infinitely extended in the horizontal directions, so that in the motionless state, the system may be treated as one-dimensional (i.e., $z$ direction only). The fluid consists of two components, with the mixing ratio being described by the mass concentration $c=m_{1} /\left(m_{1}+\right.$ $m_{2}$ ), which is assumed to be initially homogeneously distributed:

$$
c(z, t=0)=c_{0} .
$$

Apart from the ordinary mass diffusion, the fluid components are subject to an additional mass transport due to the Soret effect, resulting in a total concentration current

$$
j=-D\left[\partial_{z} c+S_{\mathrm{T}} c(1-c) \partial_{z} T\right],
$$

with $S_{\mathrm{T}}$ being the Soret coefficient and $D$ the mass diffusion coefficient $[6,35]$. The horizontal walls at $z=0, d$ are assumed to be impermeable, so that the concentration current is forced to vanish at the boundaries:

$$
\left.j\right|_{z=0}=\left.j\right|_{z=d}=0 .
$$

For the following discussion, we will assume all concentration changes to be sufficiently small compared to the initially uniform profile, so that the factor $S_{\mathrm{T}} c(1-c) \approx S_{\mathrm{T}} c_{0}\left(1-c_{0}\right)$ remains approximately constant.

When applying a temperature difference $\Delta T$ to the cell boundaries, mass will be transported along the temperature gradient, until for large times $t \gg \tau_{\mathrm{D}}=d^{2} / D$, the Soretdriven transport is just balanced by the diffusive reverse flow. In the stationary case, a total concentration difference of

$$
\Delta c=-S_{\mathrm{T}} c_{0}\left(1-c_{0}\right) \Delta T
$$

will have been achieved between the cell boundaries.

Since the thermal diffusion is typically much faster than the mass diffusion $\left(\tau_{\kappa}=d^{2} / \kappa \ll \tau_{\mathrm{D}}\right.$ due to $\left.\kappa \gg D\right)$, we may assume that on the time scale of the Soret effect, there is always a fully developed stationary temperature profile $\partial_{z} T=-\Delta T / d=$ const., independent of the applied thermal boundary conditions. The continuity equation inferred from Eq. (A2) is then reduced to a homogeneous diffusion equation

$$
\partial_{t} c=-\partial_{z} j=D \partial_{z}^{2} c,
$$

with Neumann boundary conditions enforced by the impermeable walls [Eq. (A3)]

$$
\left.\partial_{z} c\right|_{z=0}=\left.\partial_{z} c\right|_{z=d}=S_{\mathrm{T}} c_{0}\left(1-c_{0}\right) \frac{\Delta T}{d}=-\frac{\Delta c}{d}
$$

at the top and bottom boundaries [36].

To simplify the expressions, in the following discussion we will treat $\Delta c$ as the experimentally controllable parameter, which always denotes the total concentration difference that, according to Eq. (A4), would have built up in the equilibrium state, even if the system becomes unstable against convection much earlier, as discussed below.

The analytical solution to the Neumann problem stated by Eqs. (A5) and (A6) together with the initial condition (A1) can be expressed by a Fourier expansion:

$$
\begin{aligned}
c(z, t) & =c_{0}+\Delta c\left[\frac{1}{2}-\frac{z}{d}-\sum_{n=1}^{\infty} \frac{8 \cos \left(q_{n} z\right)}{(2 n-1)^{2} \pi^{2}} \mathrm{e}^{-D q_{n}^{2} t}\right] \\
\text { with } \quad q_{n} & =(2 n-1) \frac{\pi}{d} .
\end{aligned}
$$

The temporal development is shown in Fig. 11. For $t \gg \tau_{\mathrm{D}}$, the linear stationary profile according to Eq. (A4) will be achieved. Note that due to the Neumann boundary conditions (A6), the concentration gradient $\partial_{z} c$ at the boundaries is forced to its final stationary value already at the very beginning. Also note that the concentration profile in Fig. 11 is normalized to $\Delta c$. For a positive $\Delta T$ (i.e., heated from below) and a positive $S_{\mathrm{T}}, \Delta c$ is negative and the resulting concentration profile is monotonically rising.

For $t \ll \tau_{\mathrm{D}}$, we can treat the system as two independent walls with infinite distance. In this limit, Eq. (A7) can be approximated by the analytical solutions of switch-on processes at two semi-infinite half-spaces [37]. The profile near the lower boundary is then given by

$$
\begin{aligned}
c(z, t)= & c_{0}+\Delta c\left[\frac{2}{d} \sqrt{\frac{D t}{\pi}} \exp \left(\frac{-z^{2}}{4 D t}\right)-\frac{z}{d} \operatorname{erfc}\left(\frac{z}{2 \sqrt{D t}}\right)\right] \\
= & c_{0}+\Delta c\left[\frac{2}{\pi^{1 / 2}} \sqrt{\frac{t}{\tau_{\mathrm{D}}}} \exp \left(-\frac{(z / d)^{2}}{4 t / \tau_{\mathrm{D}}}\right)\right. \\
& \left.-\frac{z}{d} \operatorname{erfc}\left(\frac{z / d}{2 \sqrt{t / \tau_{\mathrm{D}}}}\right)\right], \\
\partial_{z} c(z, t)= & \frac{\Delta c}{d} \operatorname{erfc}\left(\frac{z}{2 \sqrt{D t}}\right)=\frac{\Delta c}{d} \operatorname{erfc}\left(\frac{z / d}{2 \sqrt{t / \tau_{\mathrm{D}}}}\right) .
\end{aligned}
$$

The corresponding profile near the upper wall is obtained by substituting $z \rightarrow d-z$ in Eqs. (A8) and (A9).

Following the method introduced by Howard [27], we approximate the concentration field near the boundary by a layer with a linear concentration profile

$$
c_{\text {lin }}(z, t)-c_{0}=c_{1}\left(1-\frac{z}{\delta}\right),
$$

with

$$
c_{1}=c(z=0, t)=\frac{\Delta c}{d} 2 \sqrt{\frac{D t}{\pi}}=\frac{2 \Delta c}{\pi^{1 / 2}} \sqrt{\frac{t}{\tau_{\mathrm{D}}}}
$$


being the concentration at the boundary (see Fig. 12). The slope of the profile is given by the gradient at the boundary, $\partial_{z} c(z=0, t)=-\Delta c / d$, which yields a layer height $\delta$ of

$$
\delta(t)=-\left.\frac{c_{1}}{\partial c / \partial z}\right|_{z=0}=2 \sqrt{\frac{D t}{\pi}}=\frac{2 d}{\pi^{1 / 2}} \sqrt{\frac{t}{\tau_{\mathrm{D}}}},
$$

defined by the zero-crossing of the linear profile. We now consider a "local" solutal Rayleigh number $r_{\mathrm{s}}$ calculated only over the linearized boundary layer of thickness $\delta$ :

$$
r_{\mathrm{s}}=\frac{\beta g}{D v} \delta^{3} c_{1}=\frac{\beta g}{D v} d^{3} \Delta c \frac{2^{4}}{\pi^{2}}\left(\frac{t}{\tau_{\mathrm{D}}}\right)^{2} .
$$

Dividing the local Rayleigh number by the corresponding expression for the global Rayleigh number [Eq. (1)] yields

$$
\frac{r_{\mathrm{s}}}{R_{\mathrm{s}}}=\frac{2^{4}}{\pi^{2}}\left(\frac{t}{\tau_{\mathrm{D}}}\right)^{2} .
$$

Again following Howard [27], we will assume that the boundary layer profile becomes unstable against convection once its local Rayleigh number $r_{\mathrm{s}}$ exceeds the critical value $R_{\mathrm{S}, \mathrm{c}}$, corresponding to a stationary density profile. The latency time $t_{\text {lat }}$ from switching on the temperature difference until $r_{\mathrm{s}}\left(t_{\mathrm{lat}}\right)=R_{\mathrm{s}, \mathrm{c}}$ is

$$
\frac{t_{\text {lat }}}{\tau_{\mathrm{D}}}=\underbrace{\frac{\pi}{4}}_{\approx 0.79}\left(\frac{R_{\mathrm{s}}}{R_{\mathrm{s}, \mathrm{c}}}\right)^{-1 / 2} .
$$

The "critical" height of the boundary layer at this instant of time is $\delta^{*}=\delta\left(t_{\text {lat }}\right)$ :

$$
\frac{\delta^{*}}{d}=\left(\frac{R_{\mathrm{s}}}{R_{\mathrm{s}, \mathrm{c}}}\right)^{-1 / 4} .
$$

An alternative definition for the layer height, presented by Shliomis and Souhar [29], is to require the integral over the concentration profile to be conserved:

$$
\int_{0}^{\delta} 1-\frac{z}{\delta} d z=\int_{0}^{\infty} \operatorname{erfc}\left(\frac{z}{2 \sqrt{\kappa t}}\right) d z,
$$

resulting in a layer height

$$
\delta(t)=\sqrt{\pi D t}=d \pi^{1 / 2} \sqrt{\frac{t}{\tau_{\mathrm{D}}}},
$$

and slightly different prefactors for the onset latency time and critical layer height:

$$
\begin{aligned}
& \frac{t_{\text {lat }}}{\tau_{\mathrm{D}}}=\underbrace{\frac{1}{\sqrt{2 \pi}}}_{\approx 0.40}\left(\frac{R_{\mathrm{s}}}{R_{\mathrm{s}, \mathrm{c}}}\right)^{-1 / 2}, \\
& \frac{\delta^{*}}{d}=\underbrace{\left(\frac{\pi}{2}\right)^{1 / 4}}_{\approx 1.1}\left(\frac{R_{\mathrm{s}}}{R_{\mathrm{s}, \mathrm{c}}}\right)^{-1 / 4} .
\end{aligned}
$$

[1] S. Chandrasekhar, Hydrodynamic and Hydromagnetic Stability (Oxford University Press, Oxford, UK, 1961).

[2] F. H. Busse and J. A. Whitehead, J. Fluid Mech. 47, 305 (1971).

[3] F. H. Busse, Rep. Prog. Phys. 41, 1929 (1978).

[4] M. C. Cross and P. C. Hohenberg, Rev. Mod. Phys. 65, 851 (1993).

[5] E. Bodenschatz, W. Pesch, and G. Ahlers, Annu. Rev. Fluid Mech. 32, 709 (2000).

[6] J. K. Platten and J. C. Legros, Convection in Liquids (Springer, New York, 1984).

[7] F. Winkel, S. Messlinger, W. Schöpf, I. Rehberg, M. Siebenbürger, and M. Ballauff, New J. Phys. 12, 053003 (2010).

[8] S. Rasenat, G. Hartung, B. L. Winkler, and I. Rehberg, Exp. Fluids 7, 412 (1989).

[9] W. Schöpf, J. C. Patterson, and A. M. H. Brooker, Exp. Fluids 21, 331 (1996).

[10] W. Merzkirch, Flow Visualization (Academic Press, New York, 1987).

[11] M. Ballauff and Y. Lu, Polymer 48, 1815 (2007).

[12] M. Siebenbürger, M. Fuchs, and M. Ballauff, Soft Matter 8, 4014 (2012).

[13] H. Senff, W. Richtering, C. Norhausen, A. Weiss, and M. Ballauff, Langmuir 15, 102 (1999).

[14] J. Crassous, A. Wittemann, M. Siebenbürger, M. Schrinner, M. Drechsler, and M. Ballauff, Colloid Polym. Sci. 286, 805 (2008).

[15] R. Kita and S. Wiegand, Macromolecules 38, 4554 (2005).
[16] S. Wongsuwarn, R. C. D. Vigolo, A. M. Howe, A. Vailati, R. Piazza, and P. Cicuta, Soft Matter 8, 5857 (2012).

[17] A. Königer, N. Plack, W. Köhler, M. Siebenbürger, and M. Ballauff, Soft Matter 9, 1418 (2013).

[18] B. Huke, H. Pleiner, and M. Lücke, Phys. Rev. E 75, 036203 (2007).

[19] P. Colinet, J. C. Legros, and M. G. Velarde, Nonlinear Dynamics of Surface-Tension-Driven Instabilities (Wiley-VCH, New York, 2001).

[20] D. A. Nield and A. Bejan, Convection in Porous Media, 3rd ed. (Springer, New York, 2006).

[21] IAPWS, Release on the refractive index of ordinary water substance as a function of wavelength, temperature and pressure, Available at http://www.iapws.org (1997).

[22] IAPWS, Supplementary release on properties of liquid water at $0.1 \mathrm{MPa}$, Available at http://www.iapws.org (2008).

[23] See Supplemental Material at http://link.aps.org/supplemental/ 10.1103/PhysRevE.88.053019 for video recordings of the transient convection onset.

[24] K. E. Daniels, B. B. Plapp, and E. Bodenschatz, Phys. Rev. Lett. 84, 5320 (2000).

[25] J. E. Hart, J. Fluid Mech. 47, 547 (1971).

[26] R. P. Davies-Jones, J. Fluid Mech. 44, 695 (1970).

[27] L. N. Howard, in Proceedings of the 11th International Congress of Applied Mechanics, Munich (Germany) 1964, edited by H. Görtler (Springer, New York, 1966), pp. 1109-1115.

[28] S. Messlinger, W. Schöpf, and I. Rehberg, Int. J. Heat Mass Transfer 62, 336 (2013). 
[29] M. I. Shliomis and M. Souhar, Europhys. Lett. 49, 55 (2000).

[30] D. A. Nield, J. Fluid. Mech. 71, 441 (1975).

[31] M. C. Kim, Eur. Phys. J. E 34, 27 (2011).

[32] C. K. Choi, J. H. Moon, T. J. Chung, M. C. Kim, K. H. Ahn, S.-T. Hwang, and E. J. Davis, Int. J. Heat Mass Transfer 55, 1030 (2012).

[33] R. Cerbino, S. Mazzoni, A. Vailati, and M. Giglio, Phys. Rev. Lett. 94, 064501 (2005).
[34] R. C. Nielsen and R. H. Sabersky, Int. J. Heat Mass Transfer 16, 2407 (1973).

[35] S. Wiegand, J. Phys.: Condens. Matter 16, R357 (2004).

[36] D. Hurle and E. Jakeman, J. Fluid Mech. 47, 667 (1971).

[37] J. Crank, The Mathematics of Diffusion, 2nd ed. (Oxford University Press, Oxford, UK, 1986). 